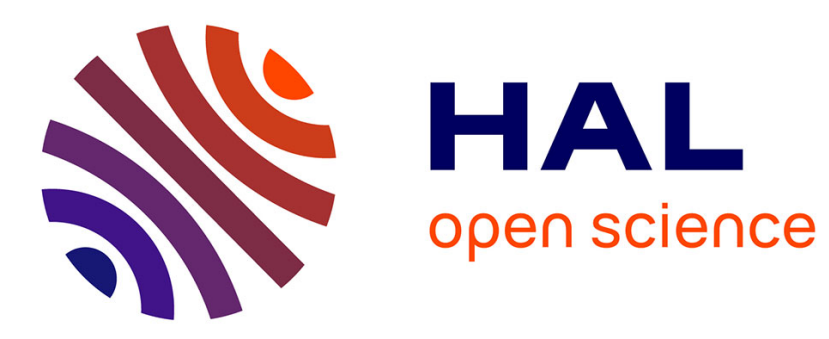

\title{
Notch signaling and the developing hair follicle
}

Geneviève Aubin-Houzelstein Aubin Houzelstein

\section{To cite this version:}

Geneviève Aubin-Houzelstein Aubin Houzelstein. Notch signaling and the developing hair follicle. Notch Signaling in Embryology and Cancer, 727, , 322 p., 2012, Advances in Experimental Medicine and Biology. hal-02811164

\section{HAL Id: hal-02811164 https://hal.inrae.fr/hal-02811164}

Submitted on 6 Jun 2020

HAL is a multi-disciplinary open access archive for the deposit and dissemination of scientific research documents, whether they are published or not. The documents may come from teaching and research institutions in France or abroad, or from public or private research centers.
L'archive ouverte pluridisciplinaire $\mathbf{H A L}$, est destinée au dépôt et à la diffusion de documents scientifiques de niveau recherche, publiés ou non, émanant des établissements d'enseignement et de recherche français ou étrangers, des laboratoires publics ou privés. 
CHAPTER 11

\title{
NOTCH SIGNALING AND THE DEVELOPING HAIR FOLLICLE
}

\author{
Geneviève Aubin-Houzelstein \\ INRA, UMR955 Génétique Fonctionnelle et Médicale; Ecole Nationale Vétérinaire d'Alfort, Maisons-Alfort, \\ France; CNRS URA2578 Département de Biologie du Développement; Institut Pasteur, Unité de Génétique \\ Fonctionnelle de la Souris, Paris, France \\ Email: ghouzelstein@vet-alfort.fr
}

\begin{abstract}
Notch function in the hair follicle has been mainly studied by use of transgenic mice carrying either loss or gain of function mutations in various members of the pathway. These studies revealed that whereas embryonic development of the hair follicle can be achieved without Notch, its postnatal development requires an intact Notch signaling in the hair bulb and the outer root sheath. Among the many roles played by Notch in the hair follicle, two can be highlighted: in the bulge, Notch controls a cell fate switch in hair follicle stem cells or their progenitors, preventing them from adopting an epidermal fate. In the hair bulb, Notch controls cell differentiation, ensuring the proper development of every layer of the hair shaft and inner root sheath. Notch function in the hair follicle is both cell autonomous and cell non autonomous and involves intercellular communication between adjacent layers.
\end{abstract}

\section{INTRODUCTION}

Hair follicles are the major appendages of the skin. They produce hairs involved in a number of functions including thermoregulation, collecting sensory information, protection against physical trauma and camouflage. Together with ist associated structures, namely the sebaceous gland, the apocrine gland and the arrector pili muscle, the hair follicle constitutes the pilosebaceous unit, a complex miniorgan of the skin. Hair follicle development occurs during embryogenesis but also throughout adult life as it is subjected to phases of growth and regression involving the cyclic recruitment of hair follicle stem cells. The development of hair follicles requires reciprocal signalings between epidermal

Notch Signaling in Embryology and Cancer, edited by Jörg Reichrath and Sandra Reichrath. (C)2012 Landes Bioscience and Springer Science+Business Media. 
and dermal compartments. In this chapter, the function of Notch signaling in the process of hair follicle development and renewal is reviewed.

\section{DEVELOPMENT AND STRUCTURE OF THE HAIR FOLLICLE}

The hair follicle has a mixed origin, as it contains cells deriving from both the epidermis and dermis. During embryogenesis, a series of reciprocal and coordinated signals between epithelial cells from the epidermis and mesenchymal cells from the dermis trigger the formation of hair follicles (Fig. 1) ${ }^{1-4}$ Hair follicle development includes three main steps: hair follicle induction, leading to the formation of hair placodes which are small invaginations of epidermis into the underlying dermis; hair follicle organogenesis, the result of which is the hair peg; and hair follicle cytodifferentiation, leading to the formation of the bulbous peg. ${ }^{4}$

The initial cues determining the position and induction of hair placodes come from the dermis. As a result, epidermal cells proliferate as small downgrowths into the underlying dermis. The main factors involved in placode formation include Fibroblast Growth Factors (FGF), Bone Morphogenetic Protein (BMP)-inhibitory factors and Wingless (Wnt) signaling molecules. Placode growth requires additional signals coming from both dermal and epidermal cells within the placode. As it grows, the embryonic hair follicle further invaginates into the dermis. Epidermal cells signal to the mesenchyme through Sonic hedgehog to form a dermal aggregate, the future dermal papilla, that is surrounded by a group of mitotically active cells, the future matrix of the hair bulb (see Figs. 2,3). The matrix contains lineage-restricted precursor cells located around the dermal papilla. Reciprocal signals between the progenitor cells in the matrix and cells in the dermal papilla lead to activation of the differentiation program for the six layers of the fully grown hair follicle, creating concentric rings of differentiated cell types. ${ }^{3-5}$ The proximodistal position of the progenitor cells within the hair matrix governs the fate of their progeny which moves upwards, exits the cell cycle and gradually expresses its differentiating program. ${ }^{6}$

The mature hair follicle undergoes phases of growth (anagen), regression (catagen) and quiescence (telogen) referred to as the hair cycle (Fig. 2). Not every part of the hair follicle is subject to morphological changes related to the phase of the cycle. Indeed, the upper third of the hair follicle does not cycle obviously and is thus known as the permanent portion, whereas the lower two-thirds, called the transitory portion, are subject to alternating phases of growth and regression depending on the phase of the cycle (Fig. 3). ${ }^{7}$

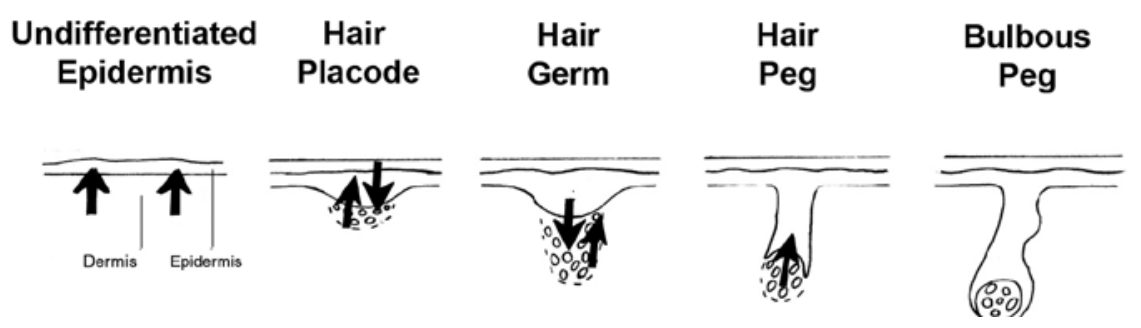

Figure 1. Embryonic development of a pelage hair follicle in the mouse. The sequential steps are indicated from left to right, with arrowheads representing the signals sent and received by the epidermal and dermal parts of the forming hair follicle. Adapted from Schneider MR et al. Curr Biol 2009; 19:R132-R142; ${ }^{4}$ C2009 with permission from Elsevier. 


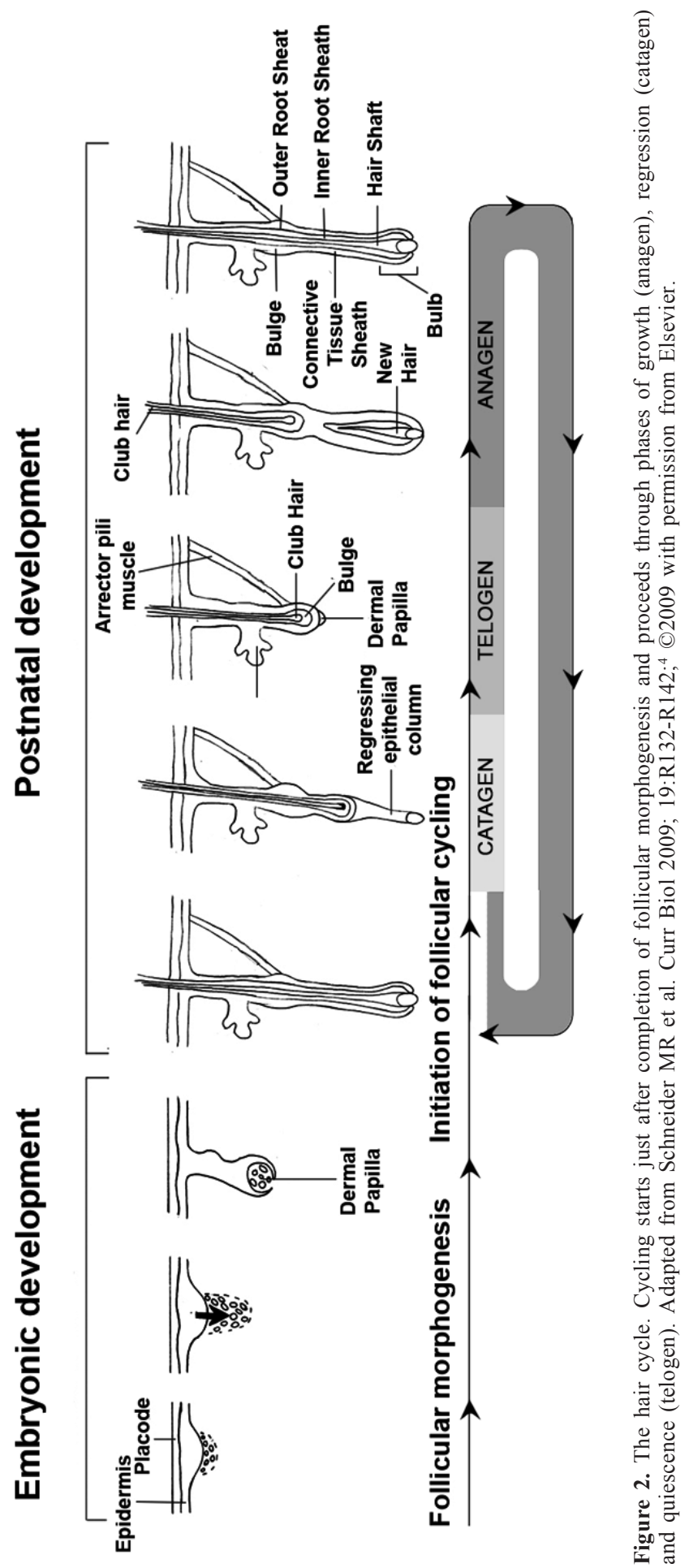




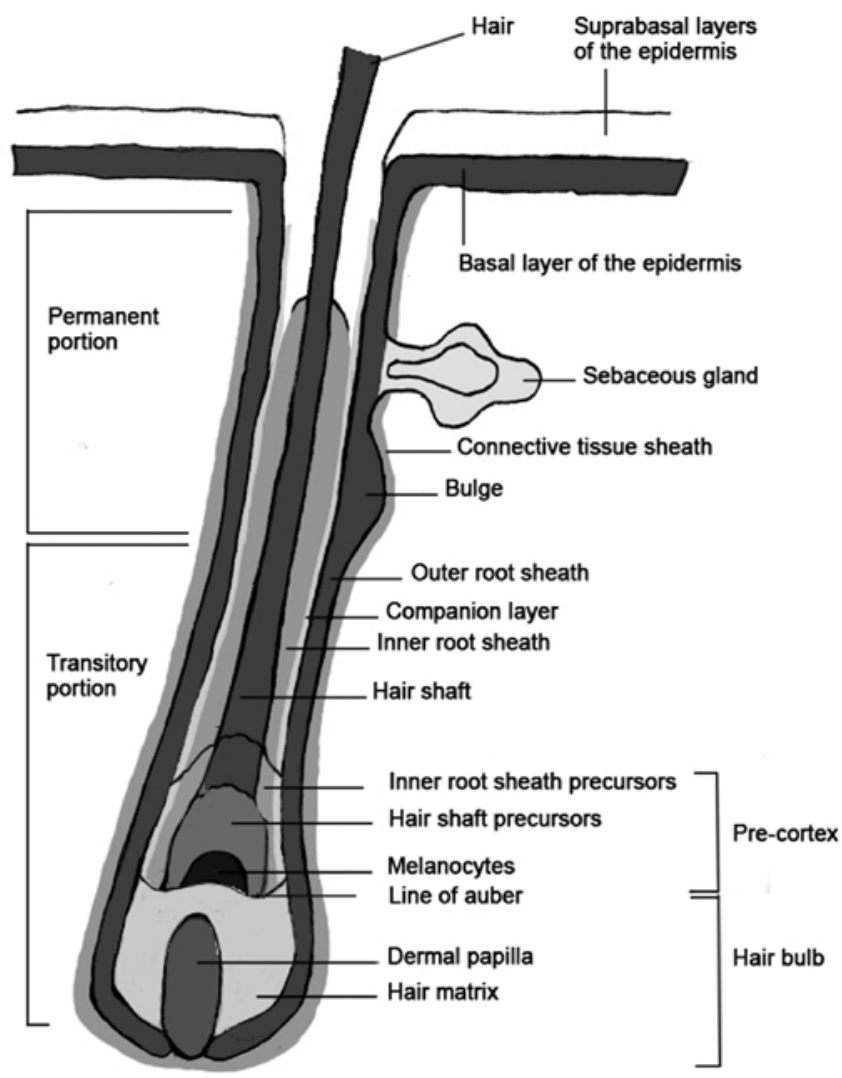

Figure 3. Structure of an anagen hair follicle in the mouse.

An anagen hair follicle is a highly organized, cylindric structure (Fig. 3) ${ }^{7,8}$ At its center, the hair shaft consists of three layers of cells, the medulla, cortex and cuticle. It is surrounded by the inner root sheath (IRS), composed of the cuticle, Huxley and Henle layers. The hair shaft and IRS are surrounded by the outer root sheath (ORS) which is a stratified epithelium, continuous with the epidermis and separated from the IRS by the companion layer. They are produced by the mitotically active cells in the matrix of the hair bulb. The rapidly proliferating cells in the matrix are located under the "line of Auber", which separates them from the cells initiating differentiation along the lineages of the IRS and hair shaft. In consequence of cell differentiation, each cell layer above the line of Auber expresses specific genes (Fig. 4). The region above the line of Auber where cells differentiate is also known as the precortex. The matrix also contains melanocytes, lying against the dermal papilla and responsible for synthesis and transport of melanin granules towards the keratinocytes of the forming hair. In the uppermost part of its permanent portion, the hair follicle is surrounded by the sebaceous gland which secretes lipids into the hair canal. Just under the sebaceous gland, the ORS presents a convex extension known as the bulge where the stem cells for keratinocytes and melanocytes are found. 


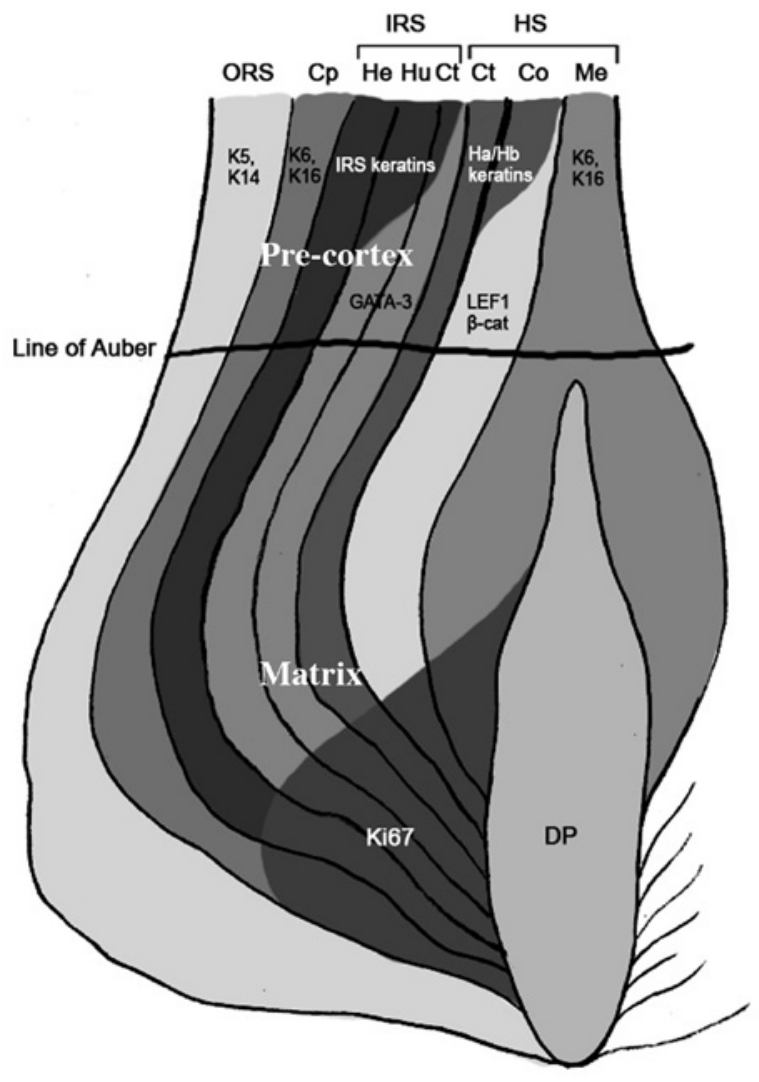

Figure 4. Genetic markers for the differented lineages in the hair follicle. The line of Auber delimits the matrix containing proliferative, Ki67 expressing cells and the precortex where cells start differentiating and expressing specific markers : Keratin 5 (K5) and Keratin 14 (K14) in the outer root sheath (ORS); Keratin 6 (K6) and keratin 16 (K16) in the companion layer (Cp) ; inner root sheath (IRS) keratins in the three IRS layers : Henle layer $(\mathrm{He})$, Huxley layer $(\mathrm{Hu})$ and cuticle $(\mathrm{Ct})$ and GATA-3 in $\mathrm{He}$ and $\mathrm{Ct}$; hair acidic and basic $(\mathrm{Ha} / \mathrm{Hb})$ keratins in the cuticle $(\mathrm{Ct})$ of the hair shaft $(\mathrm{HS})$; Lymphoid enhancer binding factor 1 (LEF1) and $\beta$-catenin ( $\beta$-cat) in the HS cortex ; K6 and K16 in the HS medulla. DP: dermal papilla. Adapted with permission from: Fuchs E. Nature 2007; 445(7130):834-42; (C)2007 Macmillan Publishers Ltd.

During catagen, the matrix cells stop proliferating and the melanocytes cease to produce pigment. Both cell types undergo massive apoptosis, leading to the degeneration and shortening of the hair follicle. The connective tissue surrounding the degenerating hair follicle shrinks in such a way that the dermal papilla is moved upwards. During catagen, a specialized structure called the club hair is formed by the ORS cells, anchoring the hair shaft to the follicle and allowing it to be retained through several subsequent hair cycles (Fig. 2).

During telogen, the hair follicle reaches its shortest size so that the dermal papilla is in contact with the hair bulge (Fig. 2). It allows exchange of signals between the dermal papilla and the hair bulge to occur, mimicking those occuring during embryogenesis, stimulating the stem cells and leading to the beginning of a new anagen. 


\section{EXPRESSION OF MEMBERS OF THE NOTCH PATHWAY IN THE HAIR FOLLICLE}

Expression of members of the Notch pathway in the hair follicle has been studied in the mouse by In Situ Hybridization, ${ }^{9-17}$ immunofluorescence microscopy and immunohistochemistry, ${ }^{10,17-24}$ by use of transgenic mice carrying a Green Fluorescent Protein reporter for Notch activity (TNR mice), ${ }^{19}$ a $\beta$-galactosidase reporter gene, ${ }^{25}$ or a genetic sensor of Notch1 proteolysis in vivo (N1IP-CRE mice). ${ }^{26}$ The expression pattern for several but not all receptors, ligands and target genes has been studied and is summarized below.

\section{Expression during Embryogenesis}

During embryogenesis, Notch1 mRNAs are found in the epidermal part of the hair placode, mostly the inner cells of the epidermal invagination, but not in its mesenchymal part. ${ }^{13,14}$ At the bulbous peg stage of development (see Fig. 1), Notch1 is expressed both at the mRNA ${ }^{14}$ and protein ${ }^{10}$ level in the hair bulb and in internal cells, but not in presumptive ORS cells.

Notch2 is not expressed in embryonic hair follicles. ${ }^{12}$

Delta1 is expressed during embryogenesis only, in the mesenchymal cells of the presumptive dermal papilla. ${ }^{14}$

Jagged 1 and 2 transcripts are found in the same locations as Notch 1 transcripts, that is in the epidermis and the epidermal parts of the forming hair follicle. ${ }^{14}$

Delta like 1 is expressed in the dermal papilla and the bulb of embryonic hair follicles, but not in adult hair follicles. ${ }^{25}$

Hes1 protein is found in the same locations as Notch1 in the hair peg at embryonic day 17 (E17): in the suprabasal core of cells forming the presumptive IRS. At P0, when the hair follicle matures, Hes 1 is also expressed in the hair shaft. ${ }^{10}$ At E16.5, Hes 1 is highly expressed in melanoblasts which also contain low amounts of Hes 5 and Heyl transcripts. ${ }^{21}$

Lunatic-fringe, Manic-fringe and Radical-fringe, encoding three secreted proteins modulating Notch signaling, are expressed in the hair follicle of the vibrissa at embryonic day 18.5 (E18.5). ${ }^{12,15}$

All these data suggest that there are at least two pairs of proteins involved in deciding where hair placodes will be formed: Notch 2 and Delta 1 in the dermis and Notch1 and Serrate 2 in the epidermis. Notch2, Delta1 and Lunatic Fringe genes may be required for dermal papilla formation, whereas Notch1, Serrate2 and Lunatic Fringe may be implicated in the patterning of the epidermal cells. ${ }^{12}$

\section{Expression during Postnatal Life}

In adult anagen hair follicles, Notch1 transcripts are found in the inner cells of the hair bulb and in the suprabasal cells of the ORS. ${ }^{14}$ Notch1 intracellular domain (NICD), which is produced after Notch1 activation, is strongly expressed in undifferentiated hair matrix cells and cells of the cortex and cuticle of the hair shaft; it is also expressed in a few cells in the ORS and in the cuticle layer of the IRS. ${ }^{23,17}$ In anagen hair follicles, Notch1 activity is detected above the line of Auber in the upper part of the hair bulb where cells undergo differentiation, in the cuticle of the IRS, in the ORS and in the dermal papilla. ${ }^{19}$ 
In adult hair follicles, subpopulations of cells with Notch1 activity are infrequently found in the bulge region where both keratinocyte and melanocyte stem cells reside. ${ }^{26}$

Notch 2 transcripts are found in the IRS precursors, distal to Notch1 and in the differentiated IRS cells. ${ }^{23}$

Notch 3 protein is expressed distally to Notch1 in the cortex of the hair shaft and in the sebaceous gland. ${ }^{23}$

The expression of Delta1 is not detected in adult hair follicles. ${ }^{14}$

Jagged 1 is expressed in the matrix, in precortex cells destined to form the hair shaft cortex and cuticle and in the suprabasal cells of the ORS above the bulb., ${ }^{9,14,19}$ Jagged1 expression and Notch activity seem to be elevated in regions of the epidermis where cells undergo commitment to terminal differentiation and thus are associated with transit amplifying cells rather than stem cells. ${ }^{19}$

Jagged 2 expression is mainly restricted to the bulb cells next to the dermal papilla and in the basal cells of the ORS.

Jagged1, Jagged2 and Notch1 are not expressed in advanced, differentiating hair shaft or IRS cells. ${ }^{14}$

RBPJk is expressed in the anagen hair follicle, including the bulge region. ${ }^{24}$

Hey1 protein is found in cortex and cuticle precursor cells of the hair shaft. ${ }^{10}$

Hes1 protein is mostly expressed in the IRS $;{ }^{10}$ its mRNA is abundant in the matrix and the precortex, is widely detected in the cortex, medulla and cuticle of the hair shaft and is expressed in a few ORS cells. ${ }^{9}$

Hey2 is expressed in dermal papilla cells and HeyL in the IRS and hair shaft, with some expression in the precortex. Hes 5 transcripts are found in the differentiating hair shaft medulla and in small, asymmetrical patches in the bulb near the line of Auber, in cells that may belong to the melanocyte lineage. ${ }^{9}$ Melanocytes express NICD, Hes1 and HeyL proteins, the three corresponding genes being overexpressed in melanocyte stem cells within the bulge compared to melanocytes in the bulb. ${ }^{27}$

Expression of Notch target genes in the precortex reflects activation of canonical Notch pathway as matrix cells get commited to terminal differentiation to form the hair shaft and the IRS. ${ }^{9}$

Figure 5 gives a summary of expression patterns of members of the Notch pathway in anagen hair follicles.

Telogen hair follicles express Jagged 1 and Hes 1 at low levels, but not Hes5, Hey2, HeyL or Heyl. ${ }^{9}$

Expression of ligands, receptors and target genes of the Notch pathway in both embryonic and postnatal hair follicles suggests a role for that pathway in the development of hair follicles during embryogenesis and postnatal life.

\section{NO ESSENTIAL ROLE FOR NOTCH IN THE INITIAL STEPS OF EMBRYONIC HAIR FOLLICLE DEVELOPMENT}

The role of Notch pathway in hair follicle development has been mainly studied by generating mouse mutants carrying either loss of function or gain of function mutations in members of the pathway that were known to be expressed in the hair follicle. ${ }^{9,10,18-21,23-25,28-31}$ As the invalidation of many genes of the Notch pathway is embryonic lethal, conditional or inducible mutants were producedj in which the targeted gene is deleted in a specific cell type or at a chosen moment. It can be achieved for instance by use of the Cre/loxP 


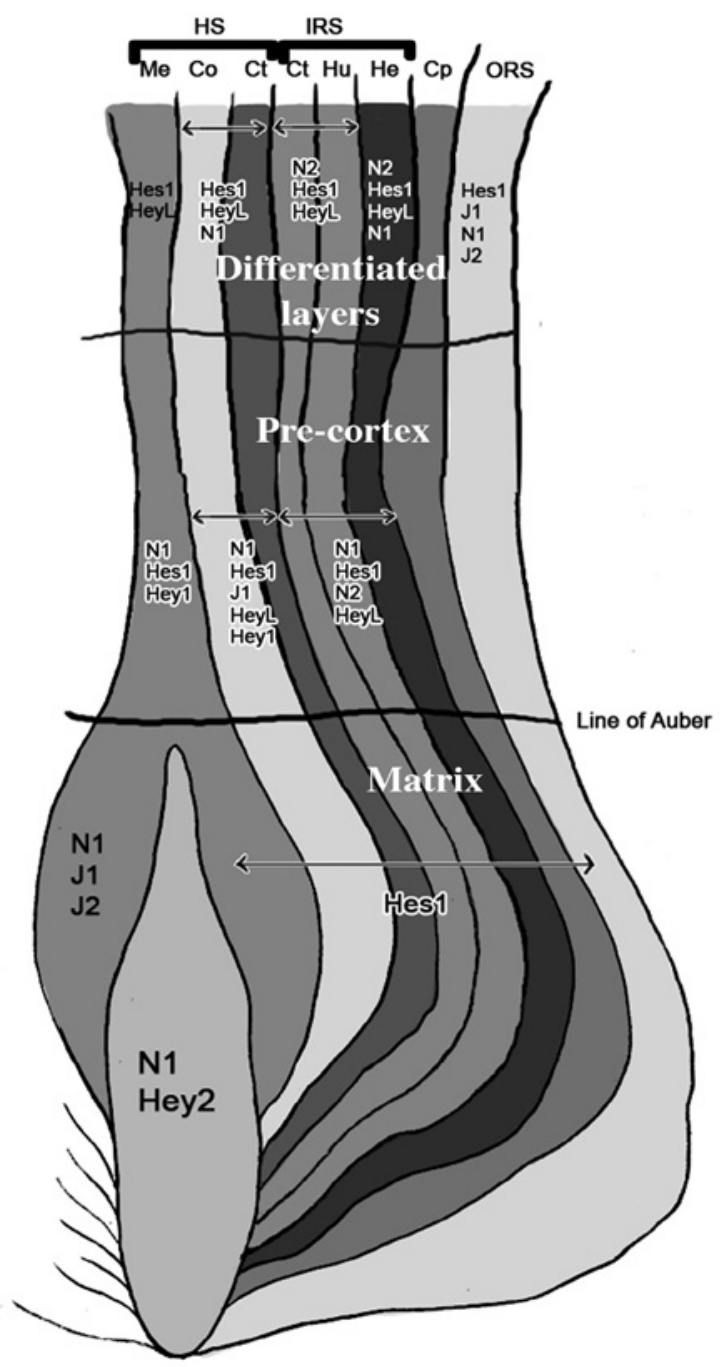

Figure 5. Expression patterns of members of the Notch pathway in an anagen hair follicle in the mouse. N1: Notch1; N2: Notch2; J1: Jagged1; J2: Jagged2; HS: hair shaft; IRS: inner root sheath; Me: medulla; Co: cortex; Ct: cuticle: Hu: Huxley layer; He: Henle layer; ORS: outer root sheath.

system for cell-specific invalidation, jointly with the tetracycline-regulated transcriptional system for inducible invalidation (see refs. 32, 33 for a review). Table 1 gives a summary of the various mutants cited in this chapter.

In every conditional knock-out for a gene in the Notch pathway in hair follicles produced so far, the number and distribution of hair follicles in the skin of newborn mutant mice are comparable to those of wild-type mice. ${ }^{10,18-20,23-25,34}$ Accordingly, in mutant mice deleted for Notchl in the basal epidermis the periodicity, spacing and the number of placodes are not altered. ${ }^{34}$ Moreover, expression patterns of signaling molecules of the 


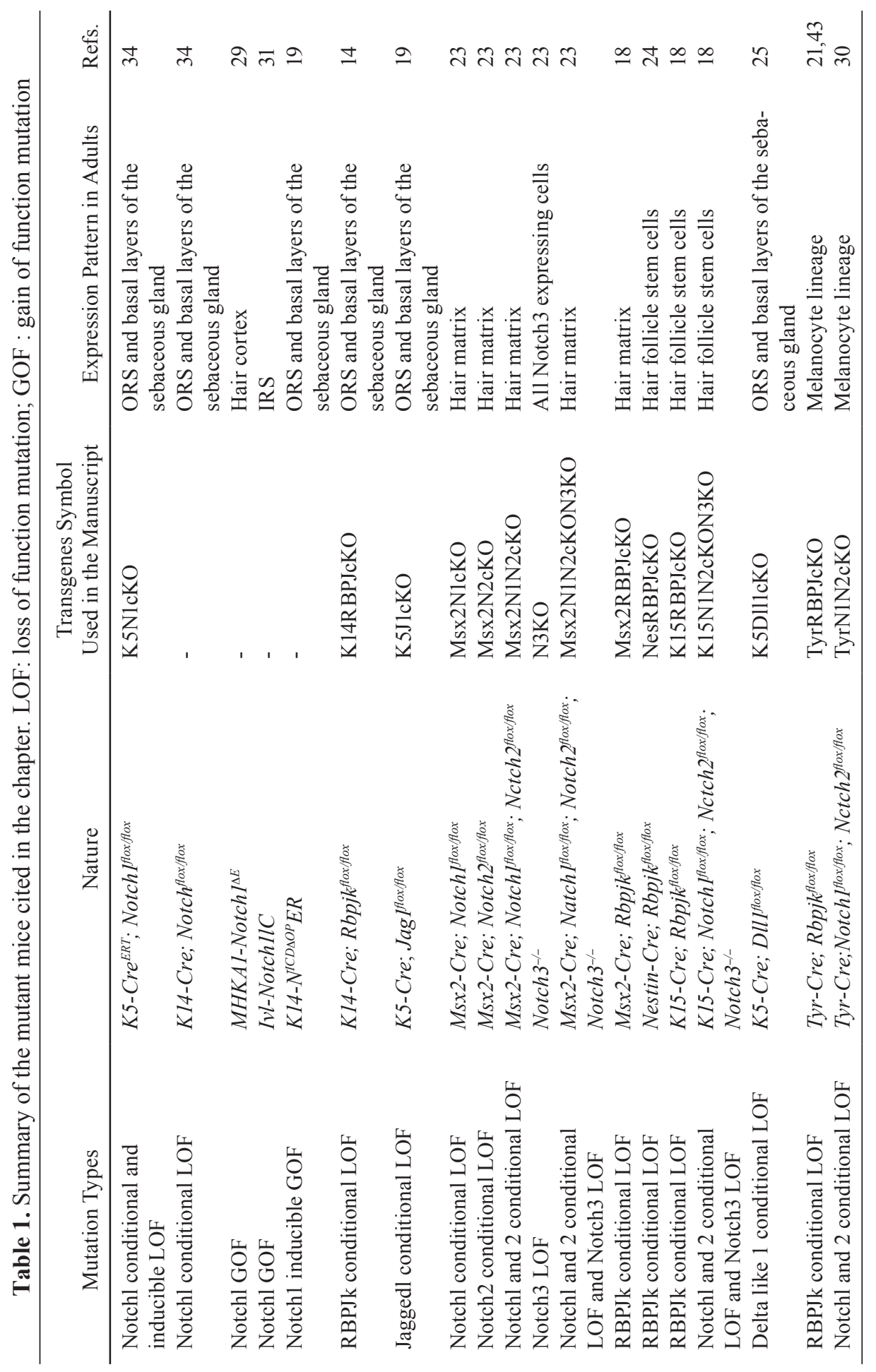


Tumor Necrosis Factor, Bone Morphogenetic Protein and Sonic hedgehog families known to be involved in early placode formation and/or in embryonic hair follicle development are not altered in the mice with a Notchl loss of function mutation. ${ }^{34}$ Thus the Notch pathway is not essential for embryonic patterning or initial specification/differentiation of hair follicles. This is in contrast to the embryonic development of feathers, where Notch plays a role in promoting feather bud development and patterning ${ }^{35}$ and in establishing the anterior-posterior asymmetry in feather buds. ${ }^{36}$

However, deletion of Notch1 in Keratin 14 expressing cells, which starts at E14.5 in the skin, is associated with an increase in hair follicle number in the dermis at E17.5 compared to littermate controls, even if at postnatal day 2 (P2), hair follicle invagination and number are normal in the mutants. ${ }^{34}$ It suggests that the placode cells invade the dermis earlier in absence of Notch1 signaling and thus that Notch1 is involved in regulating the onset of hair follicle invagination during embryogenesis.

\section{NOTCH AND THE MAINTENANCE OF CELLULAR IDENTITY WITHIN THE HAIR FOLLICLE}

Whereas Notch is not necessary for initial development of hair follicles, it plays a key role in cell differentiation within the hair follicles during late embryogenesis and adult life.

\section{Gain of Function Mutations in Notch Pathway Act Non Cell Autonomously on Hair Follicle Differentiation}

The first report of such a role for Notch describes the overexpression of an active form of Notch1 (Notch $1^{\triangle E}$ ) in precursor- and differentiated cells of the hair cortex in mouse hair keratin A1 MHKA1-Notch ${ }^{\Delta E}$ transgenic mice. ${ }^{29}$ The transgenic mice express the active form of Notch1 under the control of the MHKA1 promoter. They display curly whiskers and a shiny, wavy fur from the first anagen of the hair cycle, at P9. They start losing hairs around $\mathrm{P} 40$, at the transition between catagen and telogen during the second hair cycle and at the same stage in all the following hair cycles. In the transgenics, morphology of the IRS and ORS, hair growth and expression of IRS and hair shaft medulla markers are normal. In the upper half of the transgenic hair follicles, the cuticle is disorganized and the medulla does not contain air so that the curvature and refractory properties of the hair shaft are impaired. As MHKA1 is not expressed in either the medulla or the cuticle, it suggests that overexpression of activated Notch1 in the hair cortex precursor- and differentiated cells affects the differentiation program in the medulla and cuticle. Altered cellular interactions in the cortex may induce the expression of a secreted or surface protein, capable of altering the differentiation program in its two adjacent cell layers, the cuticle and medulla. ${ }^{29}$

Such a non autonomous function for Notch signaling has also been found in transgenic mice overexpressing a constitutively activated Notch1 in involucrin expressing cells (Ivl-Notch1IC mice). ${ }^{31}$ In these mice, the involucrin promoter drives the expression of activated Notch1 in the suprabasal layer of the interfollicular epidermis and in the IRS. In the transgenics, appearance of the first coat is delayed and the hairs are short, wavy, dull and randomly orientated. A peculiar alopecia phenotype develops from P25 as it initially preserves the midline hairs, conferring a "Mohawk-like" appearance 
to the mice. The mice are completely bald by P30 and they regrow their coat with a similar pattern in the subsequent hair cycles. In the IRS of transgenics, the cells of Henle's and Huxley's layers fail to mature and show a delay in keratinization so that no fully hardened IRS is produced. The defects in IRS differentiation include retention of nuclei within the upper portion of the IRS and the continuation of the IRS above the level of the sebaceous gland. The failure in differentiation and keratinization is more pronounced in the Henle's layer than in the Huxley's layer. This suggests that Notch signaling in IRS precursor cells controls the development of the different cell types within the IRS. However, Notch plays also a role in the differentiation of adjacent layers in the hair follicle. Indeed, the hair shafts of the Ivl-Notch1IC mice are dystrophic and display abnormalities in their three layers. Moreover, the cells of the ORS in contact with the IRS have an abnormal morphology and the ORS fails to produce club hairs. As the involucrin promoter is exclusively expressed in the IRS of the hair follicles, it is hypothesized that the defects in the hair shaft and ORS are a consequence of the abnormalities described in the IRS which makes it unable to interact with its neighbouring layers.

Alopecia may also occur when activated Notch1 is expressed in ORS cells. Indeed, overexpression of an activated form of Notch1 in Keratin 14 positive cells, including the ORS and the periphery of the sebaceous gland, has been obtained through an inducible system (K14N ${ }^{I C D} \triangle \mathrm{OP} E R$ mice). ${ }^{19}$ In the newborn mutants, the hair follicles are clumped and the hairs are not uniformly oriented. In 13 weeks mutants, the hair bulbs are enlarged and lose contact with the rest of the follicles, forming cysts consisting of an enlarged bulb surrounding an aberrant hair shaft. The cysts express markers of the companion layer, the hair bulb and the IRS, but also of terminal differentiated interfollicular epidermis. Altogether, overexpression of activated Notch1 in the ORS alters the differentiation program not only of the ORS but of several other layers in the hair follicle.

In those three models, alteration of Notch signaling was artificially obtained through either over- or ectopic expression of activated Notch1 in different layers of the hair follicle. To gain insight into the role of Notch signaling under normal conditions, several loss of function mutations were produced which ablate the signaling in hair follicle cells known to express the gene.

\section{Loss of Notch Function in Hair Follicles Leads to Alopecia and/or Alteration in the Differentiation Program}

Several loss of function mutations for members of the Notch pathway were produced in ORS cells by driving Cre expression either in Keratin 14 (K14) or K5 expressing cells. ${ }^{10,19,34}$ Keratin 5 and 14 start being expressed at E9.25 and E9.75 respectively in the basal layer of the ectoderm. ${ }^{37}$ In adult hair follicles, both K14 and K5 are expressed in the ORS and the basal layers of the sebaceous gland. ${ }^{19}$

In mice invalidated for RBPJk in K14 expressing cells (K14-Cre/+; RBPJkfoxffox (K14RBPJcKO) mice), the hair follicles are smaller and show an impaired differentiation at birth. ${ }^{10}$ In the IRS of K14RBPJcKO hair follicles at birth, fewer cells express the GATA-3 marker, specific for the IRS and the cells that express GATA-3 do so at a lower level than in wild-type hair follicles. This suggests that Notch/RBPJk signaling is required during the earliest stages of IRS differentiation. Later stages of hair follicle differentiation are also impaired: in K14RBPJcKO hair follicles in late anagen, few hair channels are formed; the remaining air channels are filled with keratins instead of air; no 
hair shaft or IRS structures can be identified. The hair follicles of K14RBPJcKO mice eventually degenerate into cystic structures.

Similarly, the loss of Notchl function in the Keratin 14 positive cells of $\mathrm{K} 14-\mathrm{Cre} /+$; Notch $1^{\text {floxffox }}(\mathrm{K} 14 \mathrm{~N} 1 \mathrm{cKO})$ mice is associated with a pronounced hair loss and a dramatic reduction in the number of hair follicles in adults. ${ }^{34} \mathrm{AtP} 13$, the remaining hairs are thinner, shorter and wavy, with an aberrant structure and the scales in the cuticle are irregular and broken. The bulb regions of the remaining hair follicles are involuted.

In mice invalidated for Jagged 1 in Keratin 5 positive cells $\left(\mathrm{K} 5-\mathrm{Cre} /+; \mathrm{Jag} \mathrm{I}^{\text {floxffox }}\right.$ $(\mathrm{K} 5 \mathrm{~J} 1 \mathrm{cKO})$ mice $),{ }^{19}$ invalidation starts at E15.5 in the basal layer of the epidermis. The $\mathrm{K} 5 \mathrm{~J} 1 \mathrm{cKO}$ mice cannot be distinguished from controls at birth but start losing their hairs and whiskers at 5 weeks and become eventually bald by 7.5 weeks, with only a few sparse, short and thin hairs left. This phenotype is very similar to what is seen in Notch1 conditional knock-out mice, suggesting that Jaggedl is the major ligand for Notch signaling in postnatal skin.

In conclusion, Notch/RBPJk signaling in the ORS plays a role in IRS/hair shaft differentiation from matrix cells.

Further insight into Notch1 function in hair follicle differentiation was gained by invalidating Notch1 in the Msx2 expressing cells of Msx2-Cre/+; Notch ffoxffox $^{-}$ $(\mathrm{Msx} 2 \mathrm{~N} 1 \mathrm{cKO})$ mice. ${ }^{23}$ In this system, Cre expression starts at E9.5 in the basal layer of the ectoderm and a second wave of Cre expression is initiated in the matrix of the hair follicles at P1. In the Msx2N1cKO mice at birth, the hairs have an altered morphology. They are wavy, finer and shorter than in controls and display bulbous alterations in the shaft. Every follicular cell type is present in the Msx2N1Msx2cKO hair follicles, but the medulla of the hair shaft and the Henle layer of the IRS are disorganized. Moreover, the expression of the medulla marker Keratin 17 is drastically reduced in the medulla of Msx2N1Msx2cKO hair follicles. Since under the line of Auber Notch1 protein is expressed in the proximal cells of the hair matrix but that above the line of Auber its expression is restricted to cortical and cuticle cells, the action of Notch1 on medulla differentiation has to be non cell autonomous. ${ }^{23}$

Although Notch 2 and Notch 3 are expressed in the hair follicle, the invalidation of either Notch 2 in the basal layer of the ectoderm in Msx2-Cre/+; Notch $2^{f t o x f f o x}(\mathrm{Msx} 2 \mathrm{~N} 2 \mathrm{cKO})$ or in Notch $^{-/-}$(N3KO) mice does not lead to any obvious skin phenotype. ${ }^{23}$ However, when both Notch1 and Notch 2 are invalidated in the basal layer of the ectoderm (Msx2N1N2cKO), the double mutants display a worse phenotype than the Msx2N1cKO single mutants, revealing some redundancy between Notch1 and Notch2 in the hair follicle. The Msx2N1N2cKO newborn have patches of naked skin that become scaly and thick. The naked regions contain hair follicles with an abnormal structure: whereas the IRS cells are initially formed, by P8, the IRS cannot be identified and is replaced by a core of pigmented, loosely packed cells, forming a degenerated hair shaft. The Msx2N1N2cKO mice die around $\mathrm{P} 25 .{ }^{23}$

The Msx2N1N2cKO phenotype is comparable to what is seen when $\gamma$-secretase is invalidated in the basal layer of the ectoderm, preventing all four Notch receptors to be activated. ${ }^{23}$ This suggests that Notch 1 and 2 are the only Notch receptors involved in hair follicle differentiation. In support to this view, removing Notch3 in an Msx2N1N2cKO background does not aggravate the hair follicle phenotype seen in Msx2N1N2cKO mice. ${ }^{23}$

It is interesting to mention that a role for noncanonical Notch signaling in hair follicle differentiation has been reported. Indeed, mice invalidated for RBPJk in Msx2 expressing cells (Msx2RBPJcKO mice) have a milder hair follicle phenotype than mice invalidated 
for Notch1 and Notch2 in Msx2 expressing cells (Msx2N1N2cKO). Furthermore, the Msx2N1N2cKO mice display the same phenotype as mice invalidated for Notch1, Notch2 and RBPJk in Msx2 expressing cells (Msx2N1N2RBPJcKO). ${ }^{18}$

Altogether, these data show that Notch is necessary for maintaining the differentiated cellular identity of the IRS, hair shaft and ORS and that its function in hair follicle differentiation is entirely mediated through Notch1 and Notch2. Notch1 and 2 act autonomously in IRS whereas Notch1 acts non-autonomously in the hair shaft.

As similar phenotypes are observed in loss- and gain of function mutants for Notch, the level of Notch signaling must be important for normal hair follicle differentiation.

\section{NOTCH AND THE REGULATION OF CELL FATE IN HAIR FOLLICLES}

One of the well-known functions for the Notch signaling pathway during mouse development is control in cell fate decisions. Such a role is fulfilled in the hair follicle. Indeed, several conditional loss of function mutations in members of the Notch pathway lead to a switch of fate in ORS cells that adopt an epidermal program. In consequence the hair follicles are replaced by epidermal cysts.

$\mathrm{K} 5 \mathrm{~J} 1 \mathrm{cKO}$ mice, invalidated for Jagged 1 in $\mathrm{K} 5$ expressing cells and $\mathrm{K} 14 \mathrm{~N}^{\mathrm{ICD} \Delta \mathrm{OP}} \mathrm{ER}$ mice overexpressing activated Notch1 in K14 expressing cells do develop epidermal cysts. ${ }^{19}$ The cysts from K5J1cKO mice are filled with cornified material. They express Keratin 10, a marker specific for interfollicular epidermis, but not markers of the IRS. On the contrary, the cysts from K $14 \mathrm{~N}^{\mathrm{ICD} \Delta \mathrm{OP}} \mathrm{ER}$ mice are expansions of the hair follicle, they resemble massive hair bulbs with aberrant hair shafts and they express several IRS markers. Whereas cysts can be the consequence of both loss and gain of Notch function, they seem to be linked to different events: a switch toward epidermal cell fate for the loss of function mutation and an aberrant differentiation for the gain of function mutation.

The study on K14N1cKO mice, invalidated for Notch1 in K14 expressing cells, supports the aberrant differentiation hypothesis. When K14N1cKO mice reach 4 weeks of age, their hair follicles are either lost or transformed into small cysts. The cysts contain a multilayered epithelium expressing Keratin 1 (K1), an early differentiation marker for interfollicular keratinocytes. ${ }^{34}$ They do not express Loricrin, a late differentiation marker for interfollicular epidermis. The authors of this study interpret cysts formation not as an impaired choice in cell fate between hair and interfollicular skin in hair follicle precursor cells. They rather think that without Notch1, the hair follicle differentiation program is altered, leading to epidermal cyst formation by default.

In Msx2N1N2cKO mice that are invalidated for both Notch1 and Notch2 in Msx2 expressing cells, initiation of catagen triggers stratification and keratinization of the ORS which eventually converts into epidermal cysts. ${ }^{23}$ In wild-type skin, ORS cells can contribute to the interfollicular epidermis only after skin injury but not under normal conditions. Thus, in wild-type mice, either the ORS cells respond to a stimulating signal produced by the wound when the skin is injured and convert into epidermal cells, or catagen hair follicles produce an inhibitory signal preventing the ORS cells to do so in the absence of skin injury. In Msx2N1N2cKO mice, the hair follicles either produce the stimulatory signal or fail to produce the inhibitory one. Whatever the reason, in absence of Notch1 and Notch2 signaling, there is a change of destiny in ORS cells which adopt an epidermal program during catagen. ${ }^{23}$ 
In these systems leading to Notch deficiency in hair follicles, the switch from a hair follicle to an epidermal differentiation program and cyst formation may have two origins: a change in cell fate in keratinocyte stem cells, or a defect in the differentiation of committed hair follicle progenitors. Whether the change of destiny occurs through a cell fate switch in bulge stem cells or through an impaired differentiation in their progenitors is not discussed in this study but the question is addressed in the two following ones: ${ }^{18,24}$

Mice invalidated for RBPJk in nestin expressing cells (nestin-Cre/+; RBPJkfoxfflox (NesRBPJcKO) mice) have a normal coat at birth but their skin displays hair loss, epidermal hyperkeratinization and cyst formation when the mice reach 1 to 3 months of age. ${ }^{24}$ The cysts express markers for all stages of epidermal differentiation, including the undifferentiation marker K5, the early differentiation markers K1 and Involucrin and the terminal differentiation markers Filaggrin and Loricrin. Differentiation within the cysts proceeds from the basal to the apical layer, with the few basal layers being highly proliferative. Hair follicle stem cells in the bulge are nestin positive and contribute to both epidermis and hair. Their progenitors destined to become epidermal cells move upwards in the upper permanent portion whereas the progenitors destined to become hair follicle cells move downwards toward the hair bulb. Tracing experiments revealed that in the hair follicles of NesRBPJcKO mice, more progenitors of the nestin positive hair follicle stem cells are found above the bulge in the upper permanent portion than in control mice. These cells elect an epidermal cell fate at the expense of the hair follicle cell fate and contribute to cyst formation. The authors of this study hypothesize that Notch/RBPJk signaling may negatively regulate the epidermal cell fate in hair follicle stem cells and facilitate their differentiation into hair cells. ${ }^{24}$

Finally, transgenic mice were produced in which canonical Notch signaling was removed specifically in bulge stem cells (K15RBPJcKO mice).$^{18}$ In these mice, deletion of $R B P J k$ is accompanied by expression of a lac $Z$ reporter gene in the deleted stem cells and their progeny, allowing cell fate mapping experiments. As expected, the K15RBPJcKO mice develop aberrant hair shafts and epidermal cysts. In these mice, $\beta$-galactosidase expressing cells are found in the hair matrix, the cortex and the cuticle of the hair shafts, proving that canonical Notch signaling is not required for hair follicle fate selection in descendents of keratinocyte stem cells. When loss of Notch1 and Notch2 function in $\mathrm{K} 15$ cells is combined with loss of Notch3 function (K15N1N2cKON3KO mice), Notch-deficient stem cells also give progeny contributing to hair follicle structures. ${ }^{18}$ Hence, neither canonical nor RBPJk-independent Notch signaling is required for stem cells to choose the follicular fate.

In both K15RBPJcKO and K15N1N1cKON3KO mice, a significant number of progeny of the deleted stem cells migrate upward from the bulge to the isthmus and epidermis where they express markers of interfollicular epidermis. ${ }^{18}$ The upward migration toward the epidermis starts before the follicles enter anagen and thus precedes the formation of aberrant hair shafts and cysts, demonstrating that improper differentiation is a consequence of the fate switch. In control mice, no cells migrate from the bulge toward the epidermis. In conclusion, a functional Notch signaling is required in hair follicle stem cells or their uncommitted descendents to prevent them from adopting the epidermal fate which, by default, restricts them to the follicular fate. 


\section{ADDITIONAL ROLES FOR NOTCH IN HAIR FOLLICLES}

\section{Control of Hair Follicle Cycling}

Transient overexpression of activated $\beta$-catenin in K14 expressing ORS cells induces telogen hair follicles to enter the hair growth cycle. This shows that $\beta$-catenin alone is able to induce anagen. ${ }^{38}$ This is not the case when an activated form of Notch1 is overexpressed also in K14 expressing cells (K14N ${ }^{\mathrm{ICD} \Delta O P} \mathrm{ER}$ transgenics); in this case, telogen hair follicles stay in the resting phase. ${ }^{19}$ Thus activation of Notch alone is not sufficient to induce anagen.

Similarly, the deletion of Notch1 in Msx2 expressing cells (Msx2N1cKO) during embryogenesis leads to shorter hair follicles the bulbs of which contain less cells than control bulbs. ${ }^{20}$ Such a phenotype might be due to an accelerated catagen onset. However, observation of hair follicle structure in Msx2N1cKO mice from P9 to P22 did not reveal any change in the timing of catagen or telogen onset.

Nevertheless, several lines of evidence show that Notch is involved in controlling hair follicle cycling:

In mice invalidated for Delta like 1 in $\mathrm{K} 5$ expressing cells (K5Dll1cKO), there is a delayed entry into the first postnatal anagen which occurs at 5 weeks in controls. ${ }^{25}$ However, subsequent hair cycles in mutants proceed at the same pace as in controls. No other defect is observed in the hair follicles of K5Dll1cKO mice.

In transgenic mice expressing the active form of Notch1 under the control of the MHKA1 promoter, the third hair cycle starts earlier (P57) than in wild-types (P89), suggesting a role for Notch in controlling the cycle clock. ${ }^{29}$

Such a role for Notch has been confirmed in mice invalidated for Notchl function in K14 or K5 expressing cells: at P9, wild-type hair follicles are in anagen and the matrix cells are actively proliferating, whereas very few proliferating cells are found in the hair matrix of Notch 1 deficient mice. ${ }^{34}$ Such a low proliferative rate is typical of the end of anagen. The lack of proliferation is not linked to an increase in apoptosis in the mutant hair matrix. In conclusion, without Notch signaling, hair follicles enter catagen prematurely. The mechanism by which Notch controls catagen entry is not known.

\section{Control of Cell Proliferation and Apoptosis in Hair Bulbs}

The hair follicles in mice invalidated for Notch1 in Msx2 expressing cells (Msx2N1cKO) during embryogenesis have smaller bulbs, containing a reduced number of cells compared to controls. ${ }^{20}$ In the mutant matrix cells at P9, mitotic rates are severely reduced, as revealed by bromodeoxyuridine (BrdU) and Ki67 labellings and apoptotic rates are slightly elevated. To investigate this phenotype, microarray analyses were performed on Msx2N1cKO hair bulbs. They revealed an elevated expression of cytostatic genes such as Cyclin kinase inhibitors $C d k n 1 a, 2 b$ and $2 c$, encoding respectively p2 $1^{\text {Cipl }}$, $\mathrm{p} 15^{\mathrm{INK} 4 \mathrm{~B}}$ and $\mathrm{p} 57^{\mathrm{Kip} 2}$ and of some proapoptotic genes such as Scotin. ${ }^{20}$

Interestingly, the expression of several Insulin Growth Factor 1 (IGF1) signaling modulators including $\operatorname{Igfbp} 2,3$ and 4 from the Insulin Growth Factor Binding Protein (IGFBP) family and of IGF receptor are elevated in Notch1-deficient hair bulbs. ${ }^{20}$ IGF1 signaling has mitogenic and anti-apoptotic activities which are antagonized by the IGFBP through their IGF1 sequestrating activity. ${ }^{39}$ In transgenic mice ectopically expressing IGFBP 3 in the involucrin expressing IRS and medulla (Ivl-Igfbp 3 mice), the hair bulbs are 
shrunk and the structure of the hair shafts is altere ${ }^{40}$ whereas overexpression of IGF1 in the IRS and medulla of $I v l$-Igfl mice triggers proliferation in the matrix.$^{41}$ These data show that IGF1::IGFBP ratio controls cell proliferation in the matrix. Combining loss of Notch1 function in Msx2 expressing cells and gain of Igfl function in Involucrin expressing cells (Msx2N1cKO; Ivl-Igfl mice) rescues the proliferation defect of Notch1-deficient matrix cells. ${ }^{20}$ Hence, the hair bulb phenotype in Msx2N1cKO Notch1 deficient mice is due to an imbalance between IGF1 and IGFBP expression. In hair bulbs, Notch1 and IGFBP2 are expressed in matrix cells whereas IGF1 and IGFBP3 are exclusively expressed in fibroblasts of the dermal papilla..$^{20,41}$ Thus in Msx2N1cKO mice, change in Notch1 expression in the hair matrix alone affects the expression of genes both in the hair matrix cells (including $I g f b p 2$ ) and in the dermal papilla (such as $I g f b p 3$ ). It reveals that both cell autonomous and non-autonomous signalings are required for proper proliferation of matrix cells and that a cross talk between two different compartments is involved in this process. ${ }^{20}$

\section{Development of Sebaceous Glands}

Invalidation of the Notch signaling pathway in hair follicles alters the differentiation of the sebaceous gland. ${ }^{10,23}$ In the skin of mice invalidated for Notch1 in Msx2 expressing cells (Msx2N1cKO mice), sebaceous glands are detected as swelling in the upper ORS at $\mathrm{P} 1$ but they are severely reduced at $\mathrm{P} 12 .{ }^{23}$ They contain few mature sebocytes, detected as Oil-Red-O and SCD1 positive cells. In Msx2NIN2cKO mutants, lacking both Notch1 and Notch2 in Msx2 expressing cells, sebaceous glands are missing. Either Notch signaling is required to set aside sebaceous progenitors or defects in sebaceous gland differentiation are a consequence of altered differentiation of the hair follicle.

Invalidation of RBPJk in K14 expressing cells severely impairs sebaceous gland differentiation since almost no Oil-Red-O staining is found in the mutant skin, revealing a dependency upon canonical Notch signaling in sebocyte differentiation. ${ }^{10}$ However, the sebaceous glands of $\mathrm{K} 5 \mathrm{~J} 1 \mathrm{cKO}$ mice, in which Jagged 1 is invalidated in $\mathrm{K} 5$ expressing cells, have a normal morphology. As both K14 and K5 are expressed in the basal layer of sebaceous glands, ${ }^{19,42}$ it suggests that Jagged 1 is not the ligand used by Notch in sebocytes.

When Notch1 is activated in K14 expressing cells (K14N ${ }^{\mathrm{ICD} \Delta O P} \mathrm{ER}$ mice), the sebaceous glands are enlarged. ${ }^{19}$ Whether the enlargement is linked to an increase in cell proliferation or in the sebocyte fate choice remains to be investigated.

\section{Melanocyte Homeostasis}

A role for Notch signaling in melanocyte biology was revealed by invalidating either RBPJk or Notch1 and Notch2 in Tyrosinase expressing cells (TyrRBPJcKO and TyrN1N2cKO mice respectively). ${ }^{21,30,43}$ In both cases, the mice had a diluted coat colour at birth, suggesting a role for Notch in the maintenance of melanocyte precursors during embryogenesis. The coat of compound mutants carrying a null allele for Notch1 or Notch 2

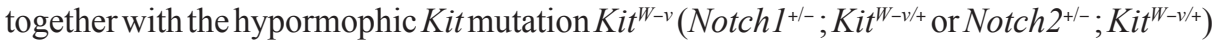
contains larger white spots than $\mathrm{Kit}^{\mathrm{W-v/+}}$ mice at birth, whereas $\mathrm{Notch}^{+/-}$and $\mathrm{Notch}^{+{ }^{+-}}$ single mutants have a coat of wild-type colour, revealing an interaction between Notch and KIT signalings in embryonic development of the melanocyte lineage. ${ }^{44}$

Strikingly, during postnatal life, the TyrRBPJcKO and TyrN1N2cKO mutants display an extensive hair graying phenotype associated with a defect in melanocyte renewal in the successive hair cycles. The number of melanocyte stem cells within the 
bulge region of mutant hair follicles dwindles with the number of hair cycles, revealing a role for Notch signaling in melanocyte stem cells maintenance. Both Notch1 and 2 are involved in the maintenance as the intensity of the hair greying phenotype depends on the number of Notch1 and/or 2 alleles deleted..$^{30}$ The maintenance defect associates apoptosis and anticipated differentiation of melanocyte stem cells and/or progenitors in the bulge region. ${ }^{21,43}$ Transcriptome analysis revealed that Notch1 and its targets Hes 1 and Heyl are overexpressed in melanocyte stem cells compared to melanocyte precursors and differentiated melanocytes. ${ }^{27}$ Accordingly, overexpression of Hes 1 in the melanocyte lineage can rescue the hair greying phenotype associated with Notch loss of function, revealing Hes 1 as the major target for Notch signaling in melanocyte stem cells. ${ }^{21}$

Notch is also involved in the control of migration and differentiation of melanocyte progenitors from the bulge to the bulb in anagen hair follicles. Indeed, in TyrRBPJcKO mice, the absence of Notch signaling is associated with the appearance of differentiated melanocytes in ectopic locations in the skin and with an impaired differentiation of melanocyte precursors within the bulb. ${ }^{43}$

Last, in mice invalidated for Notch1 in Msx2 expressing cells (Msx2N1cKO), expression in the hair bulbs of KITL, the diffusible ligand of KIT, is significantly downregulated. ${ }^{20} \mathrm{KITL}$ is known to be involved in the migration, proliferation, survival and differentiation of melanocytes and their precursors. ${ }^{45}$ Accordingly, the number of melanocytes is reduced in the hair bulbs of Msx2N1cKO mice. As melanocyte survival is dependent upon Notch-mediated activation of Hes $1,{ }^{21}$ the reduced number could be due to an altered Notch activation in melanocytes of Msx2N1cKO mice. However, Notch activation, as revealed by Hes 1 expression, is normal in the melanocytes of Msx $2 \mathrm{~N} 1 \mathrm{cKO}$ mice. Altogether, these results suggest that Notch1 activity in keratinocytes is required for proliferation and/or survival of melanocytes in the hair bulb. ${ }^{21}$

\section{CONCLUSION}

The phenotypes associated with loss and gain of function mutations in members of the Notch pathway show how tightly controlled the pathway must be in order to produce a fully developed hair follicle. It raises the question of how the expression of the genes in the Notch pathway is controlled in the hair follicle. The few data available so far deal mostly with the regulation of Notch1 expression in the hair follicle. On the one hand, Msx2 and Foxn1 proteins are required for expression of Notch1 in hair matrix cells, their expression being itself dependent on BMP2 and $4 .{ }^{17}$ On the other hand, the zinc finger protein GATA-3 is involved in downregulation of Notch 1 expression in the hair matrix. ${ }^{46}$ As GATA-3 expression is regulated through BMPs ${ }^{47}$ which are also involved in Msx 2 and Foxn1-mediated expression of Notch1, BMP signaling seems to play a central role in the control of Notch1 expression levels in the hair matrix. Finally, de novo hair follicles development after $\beta$-catenin activation in the epidermis is associated with $\beta$-catenin dependent activation of the Notch pathway in hair follicles, suggesting that Notch is placed downstream of $\beta$-catenin in hair follicle induction. ${ }^{19}$ However, further studies are needed to fully understand the control of Notch1 expression and activation in the matrix and in the bulge and to decipher how the expression of Notch ligands and Notch2 receptor is regulated in the hair follicle.

In postnatal development of the hair follicle, Notch signaling controls cell fate, proliferation, survival and differentiation not only in keratinocytes but also in melanocytes and sebocytes. Notch is involved in complex cross talks between various cell types belonging to 
several compartments in the hair follicle. ${ }^{20}$ The targets for Notch in hair follicle keratinocytes and the signals involved in the cross talks are poorly known so far. One target gene for Notch signaling in hair follicle differentiation is Desmoglein 4 (Dsg4). Indeed, the phenotype of mice invalidated for Dsg4 is similar to the phenotype of mice invalidated for Notchl and 2 in the hair follicle, in which Dsg4 expression is reduced. ${ }^{48}$ Similarly, the expression of $I g f b p 2$ and Kitl in hair matrix cells and of Igfbp 3 in dermal papilla cells is dependent on Notch signaling. ${ }^{20}$ A number of questions remain unanswered so far such as whether Dsg4, Kitl, Igfbp 2 and 3 are direct targets of Notch, what the other target genes for Notch in hair follicle are and how they exert functions as different as control of hair cycling and control of cell fate, proliferation, survival and differentiation in several cell types.

\section{ACKNOWLEDGEMENTS}

The author thanks Marc Chodkiewicz and Denis Houzelstein for critical reading of the manuscript, Gwenaëlle Salaün for artwork and the Association pour la Recherche contre le Cancer for funding (grant \#1002).

\section{REFERENCES}

1. Fuchs E. Scratching the surface of skin development. Nature 2007; 445:834-842.

2. Hardy MH. The secret life of the hair follicle. Trends Genet 1992; 8:55-61.

3. Schmidt-Ullrich R, Paus R. Molecular principles of hair follicle induction and morphogenesis. Bioessays $2005 ; 27: 247-261$.

4. Schneider MR, Schmidt-Ullrich R, Paus R. The hair follicle as a dynamic miniorgan. Curr Biol 2009; 19:R132-142.

5. Fuchs E. Beauty is skin deep: the fascinating biology of the epidermis and its appendages. Harvey Lect 1998; 94:47-77.

6. Legue E, Nicolas JF. Hair follicle renewal: organization of stem cells in the matrix and the role of stereotyped lineages and behaviors. Development 2005; 132:4143-4154.

7. Muller-Rover S, Handjiski B, van der Veen C et al. A comprehensive guide for the accurate classification of murine hair follicles in distinct hair cycle stages. J Invest Dermatol 2001; 117:3-15.

8. Paus R, Muller-Rover S, Van Der Veen C et al. A comprehensive guide for the recognition and classification of distinct stages of hair follicle morphogenesis. J Invest Dermatol 1999; 113:523-532.

9. Ambler CA, Watt FM. Expression of Notch pathway genes in mammalian epidermis and modulation by beta-catenin. Dev Dyn 2007; 236:1595-1601.

10. Blanpain C, Lowry WE, Pasolli HA et al. Canonical notch signaling functions as a commitment switch in the epidermal lineage. Genes Dev 2006; 20:3022-3035.

11. Del Amo FF, Smith DE, Swiatek PJ et al. Expression pattern of Motch, a mouse homolog of Drosophila Notch, suggests an important role in early postimplantation mouse development. Development 1992; 115:737-744.

12. Favier B, Fliniaux I, Thelu J et al. Localisation of members of the notch system and the differentiation of vibrissa hair follicles: receptors, ligands and fringe modulators. Dev Dyn 2000; 218:426-437.

13. Kopan R, Weintraub H. Mouse notch: expression in hair follicles correlates with cell fate determination. J Cell Biol 1993; 121:631-641.

14. Powell BC, Passmore EA, Nesci A et al. The Notch signalling pathway in hair growth. Mech Dev 1998; 78:189-192.

15. Thelu J, Viallet JP, Dhouailly D. Differential expression pattern of the three Fringe genes is associated with epidermal differentiation. J Invest Dermatol 1998; 111:903-906.

16. Weinmaster G, Roberts VJ, Lemke G. A homolog of Drosophila Notch expressed during mammalian development. Development 1991; 113:199-205.

17. Cai J, Lee J, Kopan R et al. Genetic interplays between Msx2 and Foxn1 are required for Notch1 expression and hair shaft differentiation. Dev Biol 2009; 326:420-430.

18. Demehri S, Kopan R. Notch signaling in bulge stem cells is not required for selection of hair follicle fate. Development 2009; 136:891-896. 
19. Estrach S, Ambler CA, Lo Celso C et al. Jagged 1 is a beta-catenin target gene required for ectopic hair follicle formation in adult epidermis. Development 2006; 133:4427-4438.

20. Lee J, Basak JM, Demehri S et al. Bi-compartmental communication contributes to the opposite proliferative behavior of Notch1-deficient hair follicle and epidermal keratinocytes. Development 2007; 134:2795-2806.

21. Moriyama M, Osawa M, Mak SS et al. Notch signaling via Hes1 transcription factor maintains survival of melanoblasts and melanocyte stem cells. J Cell Biol 2006; 173:333-339. Epub 2006 May 1.

22. Okuyama R, Nguyen BC, Talora C etal. High commitment of embryonic keratinocytes to terminal differentiation through a Notch1-caspase 3 regulatory mechanism. Dev Cell 2004; 6:551-562.

23. Pan Y, Lin MH, Tian X et al. gamma-secretase functions through Notch signaling to maintain skin appendages but is not required for their patterning or initial morphogenesis. Dev Cell 2004; 7:731-743.

24. Yamamoto N, Tanigaki K, Han $\mathrm{H}$ et al. Notch/RBP-J signaling regulates epidermis/hair fate determination of hair follicular stem cells. Curr Biol 2003; 13:333-338.

25. Estrach S, Cordes R, Hozumi K et al. Role of the Notch ligand Delta1 in embryonic and adult mouse epidermis. J Invest Dermatol 2008; 128:825-832.

26. Vooijs M, Ong CT, Hadland B et al. Mapping the consequence of Notch1 proteolysis in vivo with NIP-CRE. Development 2007; 134:535-544.

27. Nishikawa S, Osawa M. Generating quiescent stem cells. Pigment Cell Res 2007; 20:263-270.

28. Driskell RR, Giangreco A, Jensen KB et al. Sox2-positive dermal papilla cells specify hair follicle type in mammalian epidermis. Development 2009; 136:2815-2823.

29. Lin MH, Leimeister C, Gessler M et al. Activation of the Notch pathway in the hair cortex leads to aberrant differentiation of the adjacent hair-shaft layers. Development 2000; 127:2421-2432.

30. Schouwey K, Delmas V, Larue L et al. Notch1 and Notch2 receptors influence progressive hair graying in a dose-dependent manner. Dev Dyn 2007; 236:282-289.

31. Uyttendaele H, Panteleyev AA, de Berker D et al. Activation of Notch1 in the hair follicle leads to cell-fate switch and Mohawk alopecia. Differentiation 2004; 72:396-409.

32. Gossen M, Bujard H. Studying gene function in eukaryotes by conditional gene inactivation. Annu Rev Genet 2002; 36:153-173.

33. Sauer B. Inducible gene targeting in mice using the Cre/lox system. Methods 1998; 14:381-392.

34. Vauclair S, Nicolas M, Barrandon Y et al. Notch1 is essential for postnatal hair follicle development and homeostasis. Dev Biol 2005; 284:184-193.

35. Crowe R, Henrique D, Ish-Horowicz D et al. A new role for Notch and Delta in cell fate decisions: patterning the feather array. Development 1998; 125:767-775.

36. Chen CW, Jung HS, Jiang TX et al. Asymmetric expression of Notch/Delta/Serrate is associated with the anterior-posterior axis of feather buds. Dev Biol 1997; 188:181-187.

37. Lu H, Hesse M, Peters B et al. Type II keratins precede type I keratins during early embryonic development. Eur J Cell Biol 2005; 84:709-718.

38. Lo Celso C, Prowse DM, Watt FM. Transient activation of beta-catenin signalling in adult mouse epidermis is sufficient to induce new hair follicles but continuous activation is required to maintain hair follicle tumours. Development 2004; 131:1787-1799.

39. Edmondson SR, Thumiger SP, Werther GA et al. Epidermal homeostasis: the role of the growth hormone and insulin-like growth factor systems. Endocr Rev 2003; 24:737-764.

40. WegerN, Schlake T. Igfbp3 modulates cell proliferation in the hair follicle. J Invest Dermatol 2005; 125:847-849.

41. Weger N, Schlake T. Igf-I signalling controls the hair growth cycle and the differentiation of hair shafts. J Invest Dermatol 2005; 125:873-882.

42. Li L, Mignone J, Yang M et al. Nestin expression in hair follicle sheath progenitor cells. Proc Natl Acad Sci USA 2003; 100:9958-9961.

43. Aubin-Houzelstein G, Djian-Zaouche J, Bernex F et al. Melanoblasts' proper location and timed differentiation depend on Notch/RBP-J signaling in postnatal hair follicles. J Invest Dermatol 2008; 128:2686-2695.

44. Kumano K, Masuda S, Sata M et al. Both Notch1 and Notch2 contribute to the regulation of melanocyte homeostasis. Pigment Cell Melanoma Res 2008; 21:70-78.

45. Wehrle-Haller B. The role of Kit-ligand in melanocyte development and epidermal homeostasis. Pigment Cell Res 2003; 16:287-296.

46. Kurek D, Garinis GA, van Doorninck JH et al. Transcriptome and phenotypic analysis reveals GATA-3-dependent signalling pathways in murine hair follicles. Development 2007; 134:261-272.

47. Kobielak K, Pasolli HA, Alonso L et al. Defining BMP functions in the hair follicle by conditional ablation of BMP receptor IA. J Cell Biol 2003; 163:609-623.

48. Bazzi H, Demehri S, Potter CS et al. Desmoglein 4 is regulated by transcription factors implicated in hair shaft differentiation. Differentiation 2009; 78:292-300. 